\section{Calidad de vida en pacientes chilenas sobrevivientes de cáncer de mama}

\author{
M. ELISA IRARRÁZAVAL ${ }^{1}$, PASCALE KLEINMAN ${ }^{1, a}$, \\ FERNANDO SILVA R. ${ }^{3, \mathrm{~b}}$, LORETO FERNÁNDEZ GONZÁLEZ ${ }^{1, \mathrm{a}}$, \\ CAMILO TORRES ${ }^{2}$, MARCELA FRITIS ${ }^{2}$, \\ CAROLINA BARRIGA ${ }^{2}$, HERMAN WAINTRUB ${ }^{2}$
}

\section{Quality of life in Chilean breast cancer survivors}

\begin{abstract}
Background: Quality of Life (QOL) assessment may evaluate the impact of diseases and their treatment on the overall well-being of patients. Aim: To assess QOL in Chilean breast cancer survivors. Patients and Methods: Ninety one female breast cancer patients aged $60 \pm 10$ years, who finished their oncologic treatment at least a year prior to the assessment, who were disease free and in medical follow-up were included in the study. They completed the European Organization for Research and Treatment of Cancer (EORTC) QLQ-C30 core questionnaire and the breast cancer module QLQ-BR23. Results: Forty eight percent of respondents were long term survivors (more than five years). Global QOL scores were high (73.6 \pm 18.2$)$, emotional scale had the lowest scores in QLQ-C30 functional scales (72.1). Symptoms with the highest scores were: Insomnia (=21.2), pain (=20.8), and fatigue (=19.1). Body image, sexual function, and concern about the future were the most relevant problems. Body image was superior in patients with breast-conserving surgery $(p=0.008)$, and cognitive function was better in patients in early disease stage $(p=0.03)$ and in those with more than five years of survival $(p=0.04)$. Conclusions: Even when global QOL scores were high, some symptoms were prevalent. Awareness about these problems and symptoms should improve their diagnosis and treatment.
\end{abstract}

(Rev Med Chile 2016; 144: 1567-1576)

Key words: Breast Neoplasms; Quality of Life; Surveys and Questionnaires; Survivors.

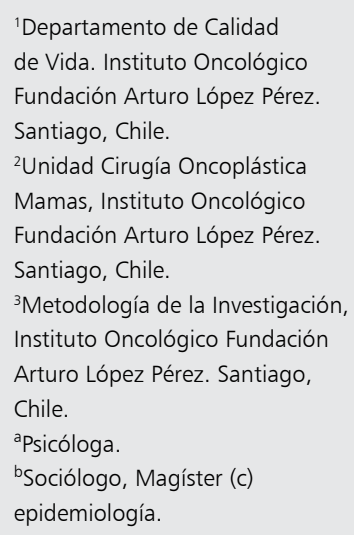

Recibido el 23 de diciembre de 2015, aceptado el 14 de octubre de 2016.

Correspondencia a: Dra. M. Elisa Irarrázaval Ibiza 5555, Vitacura marielirarrazaval@yahoo.fr
$\mathrm{E}$ n Chile, el cáncer de mama (CaM) es la primera causa de defunciones por enfermedad oncológica en la mujer ${ }^{1,2}$. La cobertura del tratamiento de CaM, incluido en las patologías GES (Garantías explícitas en Salud) desde el año 2005, ha aumentado significativamente ${ }^{3}$. Sin embargo, el envejecimiento poblacional y la prevalencia de estadios avanzados al momento de la pesquisa, no han permitido disminuir su mortalidad ${ }^{2,4}$.

La calidad de vida relacionada a la salud (CV) se define como la valoración subjetiva del paciente sobre su bienestar global, en relación a su salud y a las consecuencias de su enfermedad y tratamientos ${ }^{5-7}$. Un tratamiento exitoso debe considerar no sólo la sobrevida sino también la CV del paciente. En la práctica clínica, uno de los objetivos principales de su evaluación es optimizar las intervenciones para mejorar dicha $\mathrm{CV}$. También permite detectar grupos de mayor riesgo o vulnerabilidad, favoreciendo un abordaje interdisciplinario desde la etapa diagnóstica con intervenciones preventivas. Finalmente, la $\mathrm{CV}$ puede utilizarse con fines pronósticos que se correlaciona con la sobrevida ${ }^{7-12}$.

Las variables que afectan la CV pueden ser de tipo psicosocial o físicas. Baja escolaridad, inacti- 
vidad laboral, bajos recursos económicos, el estar sin pareja y escaso apoyo social son las variables psicosociales que disminuyen la $\mathrm{CV}$ en $\mathrm{CaM}^{7,13,14}$. Las variables físicas aumentan a mayor estadio de la enfermedad. Se ha descrito que tanto la quimioterapia (QMT) como una cirugía mayor conllevan más síntomas secundarios y peor $\mathrm{CV}^{7,13,15}$. Finalmente, se ha demostrado que la CV es más baja durante el tratamiento, buena a un año y mejor a más años de sobrevida ${ }^{11,14}$.

A la fecha, no hay ninguna publicación de CV de pacientes chilenas con CaM. El objetivo del presente estudio es describir la CV de una muestra de pacientes tratadas por $\mathrm{CaM}$, sin evidencia de enfermedad con, al menos, un año de haber concluido su tratamiento.

\section{Pacientes y Método}

En este estudio descriptivo de corte transversal, se reclutaron pacientes derivadas por sus tratantes, luego de asistir a sus controles médicos entre el 2009 y 2012.
Se incluyeron aquellas con diagnóstico histológico confirmado, mayores de 18 años, tratamiento finalizado hacía al menos un año (exceptuando la hormonoterapia como tratamiento preventivo de recidiva). Criterios de exclusión: presentar alguna discapacidad física o mental con impedimento para contestar los cuestionarios, tratamiento activo oncológico o evidencia de enfermedad. No se registraron los rechazos a participar en el estudio. Las pacientes firmaron el consentimiento informado y respondieron los cuestionarios de la EORTC (Organización Europea para la Investigación y el Tratamiento del Cáncer) QLQ-C30 (Quality of Life Questionnaire of Cancer: cuestionario general de cáncer) y QLQ-BR23 (Quality of Life Questionnaire of Breast: cuestionario módulo de cáncer de mama) (Tabla 1), validados en Chile ${ }^{16}$.

El contenidos del QLQ-C30 comprende: escalas de funcionamiento físico, rol, cognitivo, emocional y social, así como una escala de calidad de vida global; y escalas o ítems de síntomas: fatiga, náuseas y vómitos, dolor, disnea, insomnio, pérdida de apetito, estreñimiento, diarrea y un ítem

Tabla 1. Escalas de los cuestionarios de la EORTC: general para cáncer QLQ-C30 y módulo de mama QLQ-BR23

\begin{tabular}{|c|c|c|c|}
\hline & Escala & Tipo escala & Nombre abreviado \\
\hline QLQ-C30 & $\begin{array}{l}\text { Calidad de vida global } \\
\text { Funcionamiento físico } \\
\text { Funcionamiento de rol } \\
\text { Funcionamiento emocional } \\
\text { Funcionamiento cognitivo } \\
\text { Funcionamiento social } \\
\text { Cansancio } \\
\text { Náusea y vómitos } \\
\text { Dolor } \\
\text { Disnea } \\
\text { Insomnio } \\
\text { Pérdida de apetito } \\
\text { Constipación } \\
\text { Diarrea } \\
\text { Problema económico }\end{array}$ & $\begin{array}{l}\text { Salud global* } \\
\text { Funcional* } \\
\text { Funcional* } \\
\text { Funcional* } \\
\text { Funcional* } \\
\text { Funcional* } \\
\text { Síntomas** } \\
\text { Síntomas** } \\
\text { Síntomas** } \\
\text { Síntomas** } \\
\text { Síntomas** } \\
\text { Síntomas** } \\
\text { Síntomas** } \\
\text { Síntomas** } \\
\text { Síntomas** }\end{array}$ & $\begin{array}{l}\text { CVG } \\
\text { FF } \\
\text { FR } \\
\text { FE } \\
\text { FC } \\
\text { FS } \\
\text { CN } \\
\text { NV } \\
\text { DO } \\
\text { DIS } \\
\text { IN } \\
\text { PA } \\
\text { CO } \\
\text { DI } \\
\text { PE }\end{array}$ \\
\hline QLQ-BR23 & $\begin{array}{l}\text { Imagen corporal } \\
\text { Funcionamiento sexual } \\
\text { Disfrute sexual } \\
\text { Visión de futuro } \\
\text { Efectos secundarios de terapia sistémica } \\
\text { Síntomas en la mama } \\
\text { Síntomas en el brazo } \\
\text { Molestia por caída del cabello }\end{array}$ & $\begin{array}{l}\text { Funcional* } \\
\text { Funcional* } \\
\text { Funcional* } \\
\text { Funcional* } \\
\text { Síntomas** } \\
\text { Síntomas** } \\
\text { Síntomas** } \\
\text { Síntomas** }\end{array}$ & $\begin{array}{l}\text { IC } \\
\text { FSX } \\
\text { DSX } \\
\text { VF } \\
\text { ESTS } \\
\text { SM } \\
\text { SB } \\
\text { MCC }\end{array}$ \\
\hline
\end{tabular}

*A mayor puntaje en escalas funcionales mejor funcionalidad. **A mayor puntaje en escalas de síntomas mayor sintomatología. 
de impacto financiero. El QLQ-BR23 comprende escalas de imagen corporal, funcionamiento sexual, disfrute sexual y preocupación por el futuro y también: síntomas del brazo consecuencia de la cirugía, síntomas de la mama a consecuencia de la $\mathrm{RT}$, efectos secundarios del tratamiento sistémico y preocupación por la pérdida de pelo (Tabla 1).

Las escalas son tipo Likert, con puntajes de 0 , 25, 75 y 100 (nada, un poco, bastante y mucho, respectivamente). Los promedios son expresados en puntajes entre 0 y 100, para comparar con los valores de referencia (VR) publicados por la EORTC ${ }^{17}$ y otros estudios. Para las escalas funcionales, mayor puntaje significa mayor funcionalidad, pero para los síntomas, mayor puntaje indica mayor sintomatología. Las pacientes aportaron datos sociodemográficos: edad, escolaridad, situación conyugal y lugar de residencia. Se extrajeron antecedentes clínicos de las fichas: estadio de la enfermedad, tiempo transcurrido desde el diagnóstico y tratamientos recibidos. El estudio fue aprobado por el Comité de Ética del Instituto Oncológico Fundación Arturo López Pérez.

\section{Plan de análisis estadístico}

Se describió las variables de acuerdo a su escala de medición. Se hicieron pruebas no paramétricas (test U de Mann-Whitney), para definir diferencias entre grupos conocidos. Se comparó las medias de la muestra según los parámetros clínicos: estadio de la enfermedad, tipo de mastectomía (conservadora versus radical), con o sin quimioterapia, y años transcurridos desde el diagnóstico (mayor o menor a 5 años). Se corrigieron los valor de $\mathrm{p}$ con el método Bonferroni para comparaciones múltiples para las cuatro variables independientes mencionadas, se consideraron estadísticamente significativos los $\mathrm{p}<0,05$. Los análisis se realizaron en STATA 12 (.)

\section{Resultados}

\section{Descripción de la muestra}

Fueron evaluadas 103 pacientes (Tabla 2): 91 cumplieron los criterios de inclusión. Se excluyeron a 11 por estar en tratamiento o en Etapa IV. La edad fluctuó entre 38 y 83 años, con una media de $60(\mathrm{DE}=10,2)$. Sólo un poco más de la mitad (60,4\%) residía en Santiago y $2 / 3$ estaba casada o convivía. El $60 \%$ cursó estudios técnicos o universitarios, por lo que se infiere que la mayoría eran de estatus socioeconómico de nivel medio/ medio alto. Alrededor de la mitad se definieron laboralmente activas (49,5\%). El 48,4\% se había tratado hacía más de 5 años, lo cual hace de esta muestra una población con características especiales en relación a lo habitualmente publicado. En cuanto a aspectos clínicos, casi la mitad (49,5\%) se encontraban en estadios iniciales de la enfermedad. Todas las pacientes recibieron tratamiento quirúrgico: $42(46,2 \%)$ cirugía conservadora (CC) y de $49(53,8 \%)$ pacientes con mastectomía total, en $79,6 \%$ se realizó reconstrucción mamaria (MTR). Un cuarto de la muestra (24\%) fue tratada sólo con cirugía, y $45 \%$ tuvo además, radioterapia y QMT. Al menos 64,8\% de las pacientes recibió o estaba recibiendo hormonoterapia, pero no se obtuvo información en 12 de ellas.

\section{Resultados cuestionarios QLQ-C30 y QLQ-BR23}

En el QLQ-C30, la calidad de vida global (CVG) fue 73,6 (DE = 18,2), considerada alta para los VR de la EORTC (todos los puntajes allí referidos son $<64)$. Se observaron puntajes altos en todas las escalas funcionales; más de $75 \%$ de la muestra puntuó 100 en funcionamiento de rol (quehaceres y pasatiempos) y social. El funcionamiento emocional fue el más bajo $(\overline{\mathrm{X}}=72,1$ $\mathrm{DE}=24,3$ ), pero no diferente a los VR. Se observó que $25 \%$ de la muestra manifestó los siguientes problemas/síntomas: dificultades económicas, insomnio, dolor y cansancio (medias: 24,$7 ; 21,2$; 20,8 y 19,1 , respectivamente, puntajes también concordantes con los VR) (Tabla 3).

En el QLQ-BR23 (no hay VR para estos puntajes), las pacientes con alguna actividad/interés sexual fueron $44(48 \%)$, y sólo 40,9\% de estas disfrutaban bastante o mucho. En visión de futuro puntuaron $\bar{x}=16,9 / 100,(D E=17,5)$, lo que refleja alta preocupación por la salud a futuro. Si bien la mayoría de las pacientes presentó sólo síntomas leves o ninguno en la mama o brazo como secuela local quirúrgica, un cuarto de ellas sí presentó franca sintomatología, así como otro $25 \%$ presentó algún grado de sintomatología producto de terapias sistémicas -en esta muestra sólo hormonoterapia preventiva- datos no mostrados en tablas. Finalmente, "caída del cabello en la última semana" fue el síntoma de mayor relevancia, concordante con un efecto secundario de tamoxifeno. 
Tabla 2. Características sociodemográficas y clínicas de la muestra $(\mathbf{n}=91)$

\begin{tabular}{|c|c|c|c|c|}
\hline & Variable & Categorías & $\mathbf{n}$ & $\%$ \\
\hline \multirow[t]{14}{*}{ Sociodemográficas } & \multirow[t]{2}{*}{ Edad } & $<60$ & 47 & 51,7 \\
\hline & & $\geq 60$ & 44 & 48,3 \\
\hline & \multirow[t]{3}{*}{ Nivel educacional } & Escolaridad incompleta o completa & 35 & 38,5 \\
\hline & & Estudios técnicos superiores o universitarios & 55 & 60,5 \\
\hline & & Sin datos & 1 & 1,1 \\
\hline & \multirow[t]{3}{*}{ Situación ocupacional } & Sin empleo* & 44 & 48,4 \\
\hline & & Con empleo & 45 & 49,5 \\
\hline & & Sin datos & 2 & 2,2 \\
\hline & \multirow[t]{2}{*}{ Lugar de residencia } & Regiones & 36 & 39,6 \\
\hline & & Santiago & 55 & 60,4 \\
\hline & \multirow[t]{2}{*}{ Situación conyugal } & Sin Pareja & 30 & 33,0 \\
\hline & & Casada o conviviendo & 61 & 67,0 \\
\hline & \multirow[t]{2}{*}{ Años desde el diagnóstico } & Menos de 5 años & 47 & 51,6 \\
\hline & & Más de 5 años & 44 & 48,4 \\
\hline \multirow[t]{15}{*}{ Clínicas } & \multirow[t]{5}{*}{ Estadio } & $\mathrm{CIS}$ & 20 & 22,0 \\
\hline & & $\mid A$ y $\mid B$ & 25 & 27,5 \\
\hline & & $\| A$ y $\| B$ & 33 & 36,3 \\
\hline & & IIIA, IIIB y IIIC & 10 & 11,0 \\
\hline & & Sin datos** & 3 & 3,3 \\
\hline & \multirow[t]{4}{*}{ Tratamiento } & Sólo cirugía & 22 & 24 \\
\hline & & Cirugía y RT & 25 & 28 \\
\hline & & Cirugía y QT & 3 & 3 \\
\hline & & Cirugía. RT y QMT & 41 & 45 \\
\hline & \multirow[t]{3}{*}{ Tipo de mastectomía } & Cirugía conservadora & 42 & 46,2 \\
\hline & & Mastectomía total*** & 49 & 53,8 \\
\hline & & Sin datos & 3 & 3,3 \\
\hline & \multirow[t]{3}{*}{ Hormonoterapia } & No & 20 & 22 \\
\hline & & Sí & 59 & 64,8 \\
\hline & & Sin datos & 12 & 13,2 \\
\hline
\end{tabular}

$\mathrm{CIS}=$ Carcinoma in situ; RT=Radioterapia; QMT=Quimioterapia. *Incluye inactivas y/o dueñas de casa. **Pacientes diagnosticadas y tratadas en otras instituciones sin evidencia de enfermedad. ${ }^{* *}$ Un $79,6 \%$ de las mastectomías totales fue sometida a reconstrucción mamaria.

Resultados comparativos según estadio, con o sin quimioterapia y con mastectomía radical o cirugía conservadora, y tiempo transcurrido desde el diagnóstico

En pacientes con enfermedad más avanzada, se observó una tendencia a menor funcionamiento físico, debido probablemente a secuelas de tratamientos más agresivos, pero que no afectaron otras áreas $(\mathrm{p}=0,160)$, con una CVG sin diferencias. Sí hubo diferencia significativa $(\mathrm{p}=0,032)$ en cuánto a un peor funcionamiento cognitivo en estadios más avanzados (Tabla 4).

En relación a tiempo transcurrido desde el diagnóstico, a más de 5 años se observó un significativo mejor funcionamiento cognitivo $(\mathrm{p}=0,040)$ versus las diagnosticadas más recientemente (Tabla 4).

Las pacientes con MTR presentaron tendencia a menor funcionamiento de rol versus las CC $(\mathrm{p}=0,188)$, probablemente debido no sólo a secuelas quirúrgicas sino también por estar en estadios más avanzados. Las con CC presentaron significativamente $(p=0,008)$ mejor imagen corporal, y tendencia $(\mathrm{p}=0,084)$ a menos síntomas en el brazo y/o en la mama, como descrito en otros estudios $^{8,15}$ (Tabla 5). 
Calidad de vida en chilenas con cáncer de mama - M. E. Irarrázaval et al

Tabla 3. Resultados generales cuestionarios QLQ-C30 y QLQ-BR23

\begin{tabular}{|c|c|c|c|c|}
\hline & Escalas & $\mathbf{n}$ & Media & DE \\
\hline \multirow[t]{15}{*}{ QLQ-C30 } & Calidad de vida global* & 91 & 73,6 & 18,2 \\
\hline & Funcionamiento físico* & 91 & 88,9 & 11,8 \\
\hline & Funcionamiento de rol* & 91 & 89,7 & 23 \\
\hline & Funcionamiento emocional* & 91 & 72,1 & 24,3 \\
\hline & Funcionamiento cognitivo* & 91 & 84,8 & 18,6 \\
\hline & Funcionamiento social* & 91 & 86,7 & 19,6 \\
\hline & Cansancio** & 91 & 19,1 & 18,3 \\
\hline & Náusea y vómitos** & 91 & 1,1 & 4 \\
\hline & Dolor** & 91 & 20,8 & 23,1 \\
\hline & Disnea** & 91 & 8,4 & 18,8 \\
\hline & Insomnio** & 91 & 21,2 & 28,9 \\
\hline & Pérdida de apetito** & 91 & 10,5 & 22 \\
\hline & Constipación** & 91 & 17,1 & 24,8 \\
\hline & Diarrea** & 91 & 2,5 & 10,1 \\
\hline & Problema económico** & 91 & 24,7 & 30,7 \\
\hline \multirow[t]{8}{*}{ QLQ-BR23 } & Imagen corporal* & 91 & 80,5 & 25,8 \\
\hline & Funcionamiento sexual* & 90 & 56,4 & 34,8 \\
\hline & Disfrute sexual* & 91 & 17,3 & 15,1 \\
\hline & Visión de futuro* & 91 & 16,9 & 17,5 \\
\hline & Efectos secundarios de terapia sistémica** & 91 & 18,1 & 20,5 \\
\hline & Síntomas en la mama** & 37 & 18,8 & 25,3 \\
\hline & Síntomas en el brazo** & 91 & 18,6 & 19,1 \\
\hline & Molestia por caída del cabello** & 44 & 42,0 & 27,1 \\
\hline
\end{tabular}

$\mathrm{DE}=$ Desviación estándar; QLQ-C30 = Cuestionario de calidad de vida de cáncer general: QLQ-BR23=Cuestionario de calidad de vida, módulo cáncer de mama. *A mayor puntaje en escalas funcionales mejor funcionalidad. **A mayor puntaje en escalas de síntomas mayor sintomatología.

Por otra parte, sólo se observó tendencia $(\mathrm{p}=0,188)$ a un peor funcionamiento cognitivo en pacientes con QMT (Tabla 5).

\section{Discusión}

Desde la década 1970-79 existen múltiples estudios y revisiones que describen la $\mathrm{CV}$ en $\mathrm{CaM}^{11,12}$. Actualmente, los estudios se centran en las poblaciones más vulnerables o en pacientes sobrevivientes, con más de 5 años de sobrevida ${ }^{15,18,19}$. Esta población va en aumento por la mayor eficacia de los tratamientos y, por lo tanto, es de creciente interés. Nuestros resultados deben evaluarse en el contexto de pacientes con al menos un año post tratamiento, y casi la mitad con más de 5 .

La CVG, así como el funcionamiento físico, de rol y social de nuestras pacientes, fueron muy buenos en acuerdo a los estudios realizados en pacientes a al menos un año de su tratamiento y libres de enfermedad.

En este estudio se encontró que los síntomas más importantes fueron: Insomnio, dolor y cansancio. Estos han sido destacados en diversas publicaciones como importantes secuelas que 
Tabla 4. Puntajes escalas funcionales EORTC QLQ-C30 según estadio tumoral y años desde el diagnóstico

\begin{tabular}{|c|c|c|c|c|c|c|c|}
\hline \multirow[t]{2}{*}{ Escalas } & & \multicolumn{2}{|c|}{ Estadio } & \multirow[t]{2}{*}{$\mathbf{p}^{*}$} & \multicolumn{2}{|c|}{ Años desde diagnóstico } & \multirow[t]{2}{*}{$\mathbf{p}^{*}$} \\
\hline & & $\begin{array}{l}\text { CIS y I } \\
\text { (media) }\end{array}$ & $\begin{array}{l}\text { II y III } \\
\text { (media) }\end{array}$ & & $\begin{array}{c}\text { Menos de } 5 \\
(n=47) \text { (media) }\end{array}$ & $\begin{array}{c}\text { Más de } 5 \\
\text { (n = 44) (media) }\end{array}$ & \\
\hline \multirow[t]{15}{*}{ QLQ-C30 } & $\mathrm{FF}$ & 90,7 & 86,8 & 0,160 & 87,4 & 90,6 & 1 \\
\hline & $\mathrm{FR}$ & 92,2 & 87,1 & 1 & 88,2 & 91,2 & 1 \\
\hline & $\mathrm{FE}$ & 72,5 & 71 & 1 & 70,2 & 74,1 & 1 \\
\hline & $\mathrm{FC}$ & 90,2 & 83,6 & 0,032 & 80,5 & 89,2 & 0,040 \\
\hline & FS & 89,5 & 79,5 & 0,672 & 83,2 & 90,4 & 0,424 \\
\hline & CVG & 74,7 & 73,7 & 1 & 73,3 & 74 & 1 \\
\hline & $\mathrm{CN}$ & 15,9 & 22,5 & 0,244 & 23,2 & 14,8 & 0,260 \\
\hline & NV & 0,7 & 1,5 & 1 & 2 & 0 & 0,060 \\
\hline & DO & 18,6 & 23 & 1 & 19,9 & 21,7 & 1 \\
\hline & DIS & 7,3 & 10 & 1 & 9,1 & 7,5 & 1 \\
\hline & IN & 16,2 & 10 & 0,312 & 21,1 & 22,5 & 1 \\
\hline & PA & 9,5 & 11,5 & 1 & 11,3 & 9,8 & 1 \\
\hline & $\mathrm{CO}$ & 16,9 & 17,6 & 1 & 21,1 & 12,8 & 0,200 \\
\hline & $\mathrm{DI}$ & 2,9 & 1,5 & 1 & 2,1 & 3 & 1 \\
\hline & $P E$ & 18,4 & 30 & 0,420 & 24,7 & 24,8 & 1 \\
\hline \multirow[t]{8}{*}{ QLQ-BR23 } & IC & 81,3 & 80 & 0,916 & 83,1 & 77,8 & 1 \\
\hline & FSX & 15,7 & 22,6 & 0,260 & 19,2 & 18 & 1 \\
\hline & DSX & 41,3 & 44,5 & 1 & 45 & 39 & 1 \\
\hline & VF & 60,3 & 50,9 & 0,904 & 53,6 & 59,3 & 1 \\
\hline & ESTS & 15,2 & 19,7 & 0,372 & 18,6 & 15,9 & 0,780 \\
\hline & SM & 14,2 & 19,1 & 1 & 20,3 & 13,2 & 0,228 \\
\hline & SB & 18,3 & 18,4 & 1 & 18,3 & 17,5 & 1 \\
\hline & MCC & 19,5 & 18,9 & 1 & 13,2 & 22,5 & 1 \\
\hline
\end{tabular}

* $P$ values para prueba de Mann-Whitney corregidos por método Bonferroni. (Significativo $p<0,05$ ). CIS = Carcinoma in situ; QLQ-C30 = Cuestionario de calidad de vida de cáncer general; QLQ-BR23 = Cuestionario de calidad de vida, módulo cáncer de mama. FF = Funcionamiento físico; FR = Funcionamiento de rol; FE = Funcionamiento emocional; $F C=$ Funcionamiento cognitivo; FS = Funcionamiento social; CVG = Calidad de Vida global; CN = Cansancio; NV = Náusea y vómitos; DO = Dolor; $\mathrm{DIS}=$ Disnea; IN = Insomnio; PA = Pérdida de apetito; $C O=$ Constipación; $\mathrm{DI}=$ Diarrea; PE = Problema económico; IC = Imagen corporal; FSX = Funcionamiento sexual; DSX = Disfrute sexual; VF = Visión de futuro; ESTS = Efectos secundarios de terapia sistémica; SM = Síntomas en la mama; SB = Síntomas en el brazo; MCC = Molestia por caída del cabello.

presentan las pacientes a largo plazo ${ }^{7,14,20-30}$. Los estudios sobre fatiga relacionada al cáncer reportan una etiología incierta, aunque se postula un origen multifactorial, combinando condiciones derivadas de la patología y sus tratamientos, alteraciones psicológicas (depresión, ansiedad, cognitivas), y otras desregulaciones del sistema inmune $^{22-24}$. En cuanto al dolor crónico, los pacientes oncológicos presentan una mayor prevalencia que la población general, asociada a una peor CV. Es más frecuente en pacientes mayores, o sin pareja, de menores ingresos y disminuye con los años post tratamiento ${ }^{25-27}$. En relación al insomnio, síntoma frecuente en los sobrevivientes de cáncer, suele acompañarse también de dolor, ansiedad, depresión y cansancio. Su tratamiento mejora no sólo el cansancio, sino también el ánimo y la CV de las pacientes ${ }^{28,29}$. Se ha postulado que este conjunto de síntomas debiese abordarse integralmente, mas no está definido si componen un mismo síndrome o si constituyen entidades sintomáticas independientes ${ }^{30}$. Se ha destacado, también, la necesidad de un mayor apoyo a pacientes psicológicamente vulnerables, en etapas tempranas del tratamiento, además de un abordaje precoz de dichos síntomas ${ }^{22-30}$.

En relación a otros problemas, en esta muestra hubo afectación principalmente en imagen corporal, funcionamiento sexual y preocupación por el futuro. Sobre actividad y disfrute sexual, los puntajes fueron más bajos a lo publicado en general, pero similar a lo observado por Arrarás y 
Tabla 5. Puntajes escalas funcionales EORTC QLQ-C30 tipo de mastectomía y habiendo recibido o no quimioterapia

\begin{tabular}{|c|c|c|c|c|c|c|c|}
\hline \multirow[t]{2}{*}{ Escalas } & & \multicolumn{2}{|c|}{ Mastectomía } & \multirow[t]{2}{*}{ p* } & \multicolumn{2}{|c|}{ Quimioterapia } & \multirow[t]{2}{*}{$\mathbf{p}^{*}$} \\
\hline & & $\begin{array}{l}\text { Conservadora } \\
\text { ( } n=42) \\
\text { Media }\end{array}$ & $\begin{array}{c}\text { Radical } \\
(n=49) \\
\text { Media }\end{array}$ & & $\begin{array}{c}\text { Sin QT } \\
(n=45) \\
\text { Media }\end{array}$ & $\begin{array}{c}\text { Con QT } \\
(n=44) \\
\text { Media }\end{array}$ & \\
\hline \multirow[t]{15}{*}{ QLQ-C30 } & $\mathrm{FF}$ & 89,7 & 88,2 & 1 & 89,5 & 88,3 & 0,848 \\
\hline & FR & 95,6 & 84,6 & 0,188 & 91,4 & 87,8 & 1 \\
\hline & $\mathrm{FE}$ & 75,1 & 69,6 & 1 & 70,3 & 74,1 & 1 \\
\hline & $\mathrm{FC}$ & 84,6 & 84,8 & 1 & 88,5 & 80,8 & 0,188 \\
\hline & FS & 90,7 & 83,2 & 0,776 & 90,3 & 82,8 & 0,552 \\
\hline & CVG & 76,5 & 71,1 & 0,948 & 72,2 & 75,1 & 1 \\
\hline & $\mathrm{CN}$ & 17,3 & 20,7 & 1 & 16,6 & 21,8 & 0,292 \\
\hline & NV & 1,1 & 1 & 1 & 0,3 & 1,8 & 0,312 \\
\hline & DO & 16,8 & 24,2 & 1 & 18,9 & 22,8 & 1 \\
\hline & DIS & 11 & 6,1 & 1 & 7,7 & 9 & 1 \\
\hline & IN & 22 & 21,6 & 1 & 16,9 & 27,1 & 0,472 \\
\hline & PA & 6,2 & 14,2 & 0,744 & 10,6 & 10,5 & 1 \\
\hline & $\mathrm{CO}$ & 17,3 & 16,9 & 1 & 16,2 & 18 & 1 \\
\hline & $\mathrm{DI}$ & 3,2 & 2 & 1 & 2,1 & 3 & 1 \\
\hline & $\mathrm{PE}$ & 22 & 27 & 1 & 19,7 & 30,1 & 0,432 \\
\hline \multirow[t]{8}{*}{ QLQ-BR23 } & IC & 87,8 & 74,4 & 0,008 & 79,7 & 81,4 & 1 \\
\hline & FSX & 16,8 & 20,2 & 1 & 17,5 & 19,8 & 1 \\
\hline & DSX & 38,8 & 44,1 & 1 & 45 & 39 & 1 \\
\hline & VF & 63 & 50,8 & 0,648 & 59,8 & 52,7 & 1 \\
\hline & ESTS & 14,9 & 19,3 & 1 & 15,4 & 19,3 & 0,496 \\
\hline & SM & 16,4 & 17,3 & 1 & 16,4 & 17,3 & 1 \\
\hline & SB & 12,8 & 22,2 & 0,084 & 18,3 & 17,5 & 1 \\
\hline & MCC & 16,5 & 20,9 & 1 & 18,2 & 19,5 & 1 \\
\hline
\end{tabular}

* $P$ values para prueba de Mann-Whitney corregidos por método Bonferroni (Significativo $p<0,05$ ). CIS = Carcinoma in situ; QLQ-C30 = Cuestionario de calidad de vida cáncer general: QLQ-BR23 = Cuestionario de calidad de vida, módulo cáncer de mama. $\mathrm{FF}=$ Funcionamiento físico; $F R=$ Funcionamiento de rol; $F E=$ Funcionamiento emocional; $F C=$ Funcionamiento cognitivo; FS = Funcionamiento social; CVG = Calidad de Vida global; CN = Cansancio; NV = Náusea y vómitos; DO = Dolor; $\mathrm{DIS}=$ Disnea; IN = Insomnio; PA = Pérdida de apetito; CO = Constipación; DI = Diarrea; PE = Problema económico; IC = Imagen corporal; FSX = Funcionamiento sexual; DSX = Disfrute sexual; VF = Visión de futuro; ESTS = Efectos secundarios de terapia sistémica; SM = Síntomas en la mama; SB = Síntomas en el brazo; MCC = Molestia por caída del cabello.

cols. ${ }^{31}$. En estudios longitudinales se muestra una pérdida progresiva de la actividad sexual que se ha relacionado con problemas de autoimagen y a factores como sequedad vaginal y disminución de la libido, secundarios a la menopausia inducida por los tratamientos o la edad ${ }^{11,15,20,31,32}$. La importante preocupación por el futuro y las dificultades económicas se observan también en otros estudios y se relacionan al permanente temor de recidiva, a la preocupación por los hijos, o a cambios de sistema previsional ${ }^{15,31}$.

En cuanto a los tratamientos recibidos, aquí se comparó pacientes con CC versus MTR, evidenciándose tendencia a menor funcionamiento de rol, significativa peor imagen corporal y tendencia a más síntomas en el brazo en estas últimas. La evidencia cínica ha destacado que, en estadios precoces de la enfermedad, hay igual sobrevida entre CC y mastectomía total, pero con mejor $\mathrm{CV}$ en CC. La reconstrucción mamaria, si bien mejora la $\mathrm{CV}$ de las pacientes mastectomizadas, no deja de presentar mayor afectación de la CV que la cirugía conservadora ${ }^{33,34}$. La presencia de síntomas en el brazo, aun siendo sobrevivientes de $5 \mathrm{o}$ más años, está descrito por varios autores ${ }^{8,15,31,35}$, pero no parece ser suficientemente pesquisado en el seguimiento de estas pacientes. Estos podrían prevenirse o tratarse precozmente con un abordaje 
kinesiológico especializado, aunque aún no existe evidencia científica robusta al respecto ${ }^{36}$.

Los estudios indican que la QMT es el tratamiento que más afecta la $\mathrm{CV}$, incluso a largo pla$\mathrm{zo}^{8,15,31}$. En este estudio se encontró sólo una tendencia a un peor funcionamiento cognitivo de las pacientes, también referido de forma prevalente en otros estudios. El efecto de secuela neurocognitiva por la QMT se presenta con alteraciones de memoria a corto plazo, dificultades de concentración y memoria. Tiende a disminuir con el tiempo, pero persiste en un subgrupo de pacientes y debe diferenciarse de las alteraciones cognitivas por estrés y angustia transitorios asociados a tratamientos. Estos síntomas pueden impactar profundamente y cobrar importancia en la reinserción laboral ${ }^{37-38}$. No es un cuadro conocido, ni por lo tanto reconocido, en el medio oncológico chileno.

La disminución progresiva de síntomas en el tiempo, secundarios a la QMT, se corrobora aquí especialmente en el funcionamiento cognitivo, que mejora significativamente con el tiempo ${ }^{15,31}$.

Este estudio presenta las siguientes limitaciones: La muestra estudiada no necesariamente refleja la población de referencia ya que no se seleccionó los pacientes al azar sino como casos consecutivos. No se registraron las negativas a participar en el estudio, constituyendo un sesgo en la población estudiada, ya que las pacientes que participan suelen estar en mejores condiciones que aquellas que se niegan. Otra limitación es el pequeño tamaño de la muestra que, sumado a la heterogeneidad, no permitió un análisis más profundo de los datos, como, por ejemplo, la comparación por variables socioeconómicas. Tampoco existe un estudio con QLQ-C30 en población general chilena para poder comparar ciertos resultados, por ejemplo, lo referente a lo observado en sexualidad. Finalmente, los resultados de función cognitiva no son fiables ya que en la validación del QLQ-BR23 esta escala fue la única que no fue internamente consistente ${ }^{16}$.

Pese a esto, nuestros resultados en general concuerdan con los VR de la EORTC o con la bibliografía consultada.

\section{Conclusiones}

Pese a las limitaciones expuestas, este es el primer estudio que muestra la CV de pacientes chilenas, en este caso clínicamente estables, tratadas por CaM, pudiendo mostrar que los hallazgos son similares (aunque más escuetos) a lo publicado en otros países.

Se puede constatar que, pese a que la CVG fue buena, los síntomas asociados a las terapias siguen afectando, al menos, a un cuarto de las pacientes. Los síntomas que más se presentaron son: cansancio, dolor e insomnio. Estos parecen estar correlacionados entre sí y requieren una pesquisa precoz, un manejo integral, incluyendo síntomas afectivos. Por otra parte, la mastectomía total, con o sin reconstrucción, dejó secuelas a largo plazo en principalmente en imagen corporal. A estadios más avanzados se presenta peor funcionamiento cognitivo, y a mayor tiempo desde el diagnóstico éste es mejor. Finalmente, es necesario considerar las preocupaciones psicosociales reportadas en etapa de seguimiento. Futuros estudios en $\mathrm{CV}$ en Chile debiesen tener en cuenta la incorporación de dimensiones como espiritualidad, terapias complementarias y reinserción laboral de las pacientes.

A la luz de estos resultados, parece fundamental la pesquisa y manejo de síntomas residuales de los tratamientos en pacientes con CaM libres de enfermedad, así como la implementación de estrategias de abordaje interdisciplinario para optimizar la CV de estas mujeres, población cada día más numerosa.

\section{Referencias}

1. Ministerio de Salud. (s.f.). Departamento de estadísticas e informaciones en salud. Disponible en http://deis.minsal.cl/vitales/defunciones_serie/Defunciones_Mortalidad_Tumores_Malignos_2000-2009. htm. [Consultado el 14 de mayo de 2015].

2. Serra I, Martínez R, Reyes G, Sierra P, Aguayo C. Envejecimiento y alta prevalencia de estadios avanzados determinan la creciente mortalidad por cáncer mamario en Chile. Rev Chil Cir 2012; 64 (2): 137-46.

3. Ministerio de Salud. Guía Clínica cáncer de mama en personas de 15 años y más. 2005. 1st Ed. Santiago. Disponible en http://www.redsalud.gov.cl/archivos/ guiasges/CancermamaR_Mayo 10-pdf [Consultado el 14 de mayo de 2015].

4. Serra I. Cáncer de mama en Chile: pasado, presente y futuro. Rev Chil Cir 2011; 63 (6): 553-6.

5. Salas C. Calidad de vida y factores asociados en mujeres con cáncer de mama en Antioquia, Colombia. Revista Panam Salud Pública 2010; 28 (1): 9-18. 
6. Arrarás JI. Progresos en la evaluación de la calidad de vida en el cáncer de mama. El sistema de medida de la EORTC. Psicooncología 2007; 4 (2-3): 367-84.

7. Smith AW, Alfano CM, Reeve BB, Irwin ML, Bernstein L, Baumgartner K, et al. Race/ethnicity, physical activity, and quality of life in breast cancer survivors. Cancer Epidemiol Biomarkers Prev 2009; 18 (2): 656-63.

8. Montazeri A. Quality of life data as prognostic indicators of survival in cancer patients: an overview of the literature from 1982 to 2008 . Health Qual Life Outcomes 2009; 7:102. doi: 10.1186/1477-7525-7-102. Disponible en: http://www.ncbi.nlm.nih.gov/pmc/articles/ PMC2805623/ [Consultado el 14 de mayo de 2015].

9. Perry S, Kowalski T, Chang C-H. Quality of life assessment in women with breast cancer: benefits, acceptability and utilization. Health Qual Life Outcomes 2007, 2: 5-24.

10. Sarna L, Padilla G, Holmes C, Tashkin D, Brecht ML, Evangelista L. Quality of life of long-term survivors of non-small-cell lung cancer. J Clin Oncol 2002; 20 (13): 2920-9.

11. Montazeri A. Health-related quality of life in breast cancer patients: a bibliographic review of the literature from 1974 to 2007. J Exp Clin Cancer Res 2008; 29: 27-32.

12. Lemieux J, Goodwin P, Bordeleau L, Lauzier S, Théberge V. Quality-of-life measurement in randomized clinical trials in breast cancer: an updated systematic review (2001-2009). J Natl Cancer Inst 2011; 103 (3): 178-231.

13. Bajuk L, Reich M. Calidad de vida relacionada con la salud en mujeres uruguayas con cáncer de mama. Ciencias Psicológicas 2011; V (1): 19-30.

14. Ganz PA, Desmond KA, Leedham B, Rowland JH, Meyerowitz BE, Belin TR. Quality of life in long-term, disease-free survivors of breast cancer: a follow-up study. J Natl Cancer Inst 2002; 94 (1): 39-49.

15. Buijs C, Rodenhuis S, Seynaeve CM, van Hoesel QGCM, van der Wall E, Smit WJM, et al. Prospective study of long-term impact of adjuvant high dose and conventional-dose chemotherapy on health related quality of life. J Clin Oncol 2007; 25 (34): 5403-9.

16. Irarrázaval ME, Rodríguez PF, Fasce G, Silva F, Waintrub $\mathrm{H}$, Torres $\mathrm{C}$, et al. Calidad de vida en cáncer de mama: Validación del cuestionario QLQ-BR23 en Chile. Rev Med Chile 2013; 141: 723-34.

17. Scott N, Fayers P, Aaronson N, Bottomley A, de Graeff A, Groenvold M, et al. EORTC QLQ-C30. Reference values. Brussels: EORTC, 2008. p. Disponible en: http:// groups.eortc.be/qol/sites/default/files/img/newsletter/ reference_values_manual2008.pdf [Consultado el 15 de mayo de 2016].

18. Ganz PA, Kwan L, Stanton AL, Bower JE and Belin TR.
Physical and psychosocial recovery in the year after primary treatment of breast cancer. J Clin Oncol 2011; 29 (9): 1101-9.

19. Mols F, Vingerhoets AJ, Coebergh JW, van de Poll-Franse LV. Quality of life among long-term breast cancer survivors: a systematic review. Eur J Cancer 2005; 41 (17): 2613-9.

20. Avis NE, Ip E, Foley KL. Evaluation of the quality of life in adult cancer survivors (QLACS) scale for long-term cancer survivors in a sample of breast cancer survivors. Health Qual Life Outcomes 2006; 1 (4): 92.

21. Sohl SJ, Levine B, Avis NE. Evaluation of the quality of life in adult cancer survivors (QLACS) scale for early post-treatment breast cancer survivors. Qual Life Res 2015; 24 (1): 205-12.

22. Berger AM, Gerber LH, Mayer DK. Cancer-related fatigue: implications for breast cancer survivors. Cancer 2012; 118 (8 suppl): 2261-9.

23. Garabeli Cavalli Kluthcovsky AC, Urbanetz AA, de Carvalho DS, Pereira Maluf EM, Schlickmann Sylvestre GC, Bonatto Hatschbach SB. Fatigue after treatment in breast cancer survivors: prevalence, determinants and impact on health-related quality of life. Support Care Cancer 2012; 20 (8): 1901-9.

24. Yennurajalingam $S$ and Bruera E. Review of Clinical Trials of Pharmacologic Interventions for Cancer-Related Fatigue. Cancer Journal 2014; 20 (5): 319-24.

25. Casso D, Buist DS, Taplin S. Quality of life of 5-10 year breast cancer survivors diagnosed between age 40 and 49. Health Qual Life Outcomes 2004; 18 (2): 25.

26. Peuckmann V, Ekholm O, Rasmussen NK, Groenvold M, Christiansen P, Møller S, et al. Chronic pain and other sequelae in long-term breast cancer survivors: nationwide survey in Denmark. Eur J Pain 2009; 13 (5): 478-85.

27. Schreiber KL, Kehlet H, Belfer I, Edwards RR. Predicting, preventing and managing persistent pain after breast cancer surgery: the importance of psychosocial factors. Pain Manag 2014; 4 (6): 445-59.

28. Davis PD, Goforth HW. Long-term and short-term effects of insomnia in cancer and effective interventions. Cancer Journal 2014; 20 (5): 330-44.

29. Denlinger CS, Ligibel JA, Are M, Baker KS, Demark-Wahnefried W, Friedman DL, et al. National comprehensive cancer network. Survivorship: sleep disorders, version 1. 2014. J Natl Compr Canc Netw 2014; 12 (5): 630-42.

30. Zucca AC, Boyes AW, Linden W, Girgis A. All's well that ends well? Quality of life and physical symptom clusters in long-term cancer survivors across cancer types. J Pain Symptom Manage 2012; 43 (4): 720-31. 
31. Arraras J, Illarramendi J, Manterola A, Tejedor M, Vera R, Valerdi J, et al. Evaluación de la calidad de vida a largo plazo en pacientes con cáncer de mama en estadios iniciales mediante los cuestionarios de la EORTC. Rev Clin Esp 2003; 203: 577-81.

32. Pinto AC, de Azambuja E. Improving quality of life after breast cancer: dealing with symptoms. Maturitas. 2011; 70(4):343-8. doi: 10.1016/j.maturitas.2011.09.008.

33. Katz SJ, Hawley ST. From policy to patients and back: surgical treatment decision making for patients with breast cancer. Health Affairs 2007; 26 (3): 761-9.

34. Rocco N, Catanuto G, Nava MB. What is the evidence behind conservative mastectomies? Gland Surg 2015; 4 (6): 506-18.

35. Collins KK, Liu Y, Schootman M, Aft R, Yan Y, Dean $G$, et al. Effects of breast cancer surgery and surgical side effects on body image over time. Breast Cancer Res Treat 2011; 126 (1): 167-76.
36. Stuiver MM, ten Tusscher MR, Agasi-Idenburg CS, Lucas C, Aaronson NK, Bossuyt PMM. Conservative interventions for preventing clinically detectable upper-limb lymphoedema in patients who are at risk of developing lymphoedema after breast cancer therapy. Cochrane Database of Systematic Reviews 2015, Issue 2. Art. No.: CD009765. DOI: 10.1002/14651858. CD009765.pub2. Disponible en http://www.cochrane. org/CD009765/BREASTCA_interventions-for-preventing-lymphoedema-swelling-of-the-arm-after-breast-cancer-treatment [Consultado el 6 de diciembre de 2015].

37. Moore HC. An overview of chemotherapy-related cognitive dysfunction or 'chemobrain'. Oncology 2014; 28 (9): 797-804.

38. Dutta V. Chemotherapy, neurotoxicity, and cognitive changes in breast cancer. J Cancer Res Ther 2011; 7 (3): 264-9. 